\title{
Attenuation of methylation of catecholamines and treatment with a coenzyme of catechol-O-methyltransferase in spontaneously hypertensive rats (SHR)
}

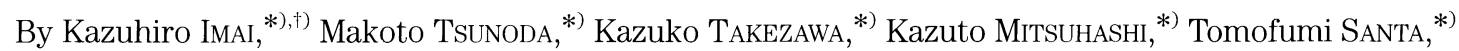 \\ Ken Nagashima, ${ }^{* *)}$ Keishi Katayama, $^{* *)}$ and Kenji Ohmori ${ }^{* *)}$ \\ (Communicated by Masanori OTsuKA, M. J. A., Sept. 13, 1999)
}

\begin{abstract}
We have previously demonstrated that the release of plasma norepinephrine (NE) in response to blood pressure reduction induced by calcium antagonist was substantially decreased in spontaneously hypertensive rats (SHR), as compared to the age-matched normotensive Wistar Kyoto (WKY) rats. Here we examined extraneuronal methylation of NE to normetanephrine (NMN) by catechol-O-methyltransferase (COMT). In the face of an acute hypotension by benidipine, the ratio of the increase of NMN to the increase of NE in plasma was lower in SHR than in WKY rat. The finding suggests that the extraneuronal inactivation of the released NE is blunted in SHR, namely, COMT is deactivated in this hypertensive rats. To define the role of NE methylation in hypertension, S-adenosyl-L-methionine (SAMe; $1.0 \mathrm{mg} / \mathrm{kg}$, i.v.), a coenzyme of COMT, was administered to SHR. The treatment induced a greater antihypertensive effect, along with a greater decrease of NE and a larger increase of NMN in SHR. The data suggest that SAMe may be used as a possible remedy for hypertension.
\end{abstract}

Key words: $\quad$ Norepinephrine; catechol-O-methyltransferase; blood pressure; S-adenosyl-L-methionine; hypertension.

Introduction. To understand the function of homeostatic systems in animals, the behavior of related biomolecules can be studied. ${ }^{1)}$ The blood pressure regulation in rats, for example, can be a representative of such a system. When the blood pressure is reduced, the first reflex by the homeostatic system is, via the sympathetic baroreflex, to induce a release of neurotransmitter norepinephrine (NE) from the nerve endings. The release is directly related to the degree of the acute hypotension: the greater the hypotensive effect, the greater the amount of NE is released. ${ }^{2), 3)}$ Previously, we demonstrated that the ratio of the increase of plasma $\mathrm{NE}$ concentration to the reduction of blood pressure was attenuated in spontaneously hypertensive rats (SHR) as compared to the age-matched normotensive Wistar Kyoto (WKY) rats. ${ }^{4), 5)}$

\footnotetext{
*) Graduate School of Pharmaceutical Sciences, The University of Tokyo, 7-3-1 Hongo, Bunkyo-ku, Tokyo 113-0033, Japan.

**) Drug Development Research Laboratories, Pharmaceutical Research Institute, Kyowa Hakko Kogyo Co., Ltd., 1188 Shimotogari, Nagaizumi-cho, Sunto-gun, Shizuoka 411-8731, Japan.

†) Correspondence to: K. Imai.
}

From this finding, we posed a fundamental question: Is the metabolism of $\mathrm{NE}$ different in the two strains? To solve this, in this paper, benidipine, a calcium antagonist, was administered to SHR and WKY rats to induce a release of $\mathrm{NE}$ via the sympathetic baroreflex as mentioned above. Then, the released NE is extraneuronally inactivated to its O-methyl metabolite (normetanephrine; NMN) by catechol-O-methyltransferase (COMT). Since the ratio of plasma NMN to plasma $\mathrm{NE}$ was thought to be a parameter for the metabolic inactivation, we simultaneously measured plasma $\mathrm{NE}$ and NMN concentration. Consequently, we found that COMT was deactivated in SHR. So that the activation of COMT was thought to bring about the blood pressure reduction in SHR. Now we selected S-adenosyl-Lmethionine (SAMe), a coenzyme of COMT, and SAMe was administered to SHR to clarify an antihypertensive effect.

Materials and method. Reagents. NE, NMN and SAMe were purchased from Sigma (St. Louis, MO, U.S.A.). Benidipine hydrochloride and heparine sodium salt were from Kyowa Hakko Kogyo Co., Ltd. (Tokyo, Japan) 
Table I. Plasma norepinephrine (NE) and normetanephrine (NMN) concentration and ratio of NMN to NE in an untreated state

\begin{tabular}{lcr}
\hline & WKY $(\mathrm{n}=5)$ & SHR $(\mathrm{n}=5)$ \\
\hline Plasma NE conc. (fmol/ml) & $507 \pm 62$ & $1,190 \pm 110^{\sharp}$ \\
Plasma NMN conc. (fmol/ml) & $273 \pm 52$ & $513 \pm 48^{*, a}$ \\
Ratio of NMN to NE & $0.53 \pm 0.04$ & $0.45 \pm 0.06$ \\
\hline
\end{tabular}

The values represent the mean \pm SEM of 5 rats per group.

${ }^{*}: \mathrm{p}<0.05,{ }^{\#}: \mathrm{p}<0.01$ vs. the values for WKY rats. ${ }^{\mathrm{a}}$ : This result is in agreement with the observation by Vlachakis et al., ${ }^{12)}$ but the magnitude of values were different. Sixteen 28-week-old SHR had a concentration of NMN of $10.0 \pm 2.6 \mathrm{pmol} / \mathrm{ml}$ while the age matched WKY rats had a value of $2.9 \pm 0.6 \mathrm{pmol} / \mathrm{ml}$ $(\mathrm{p}<0.02, \mathrm{n}=11)$.

Erythrocyte COMT activity measurement. Blood samples (10 ml each) collected in heparinized tubes from five anesthetized SHR and WKY rats (male, 23 week-old, Nihon Charles River, Kanagawa, Japan) with diethyl ether were centrifuged at 3,000 g for $10 \mathrm{~min}$ at $4^{\circ} \mathrm{C}$. Treatment of the packed red cells obtained and COMT assay procedure were performed according to the report ${ }^{6)}$ except NE as a substrate instead of 3,4-dihydroxybenzoic acid. Formed NMN was determined according to the previous report. ${ }^{\text {.) }}$

Animal experiment and analysis of $N E$ and NMN in plasma after administration of benidipine. Five SHR and WKY rats (male, 20-23 week-old) were anesthetized with pentobarbital (50 $\mathrm{mg} / \mathrm{kg}$, i.p.) and the right femoral artery and the jugular vein were catheterized with each polyethylene cannulae (PE-50) (Japan Becton Dickinson Co., Tokyo, Japan). Arterial blood pressure and heart rate were measured through the right femoral artery with cardiac-tachometer (AT601G, AP621G and Thermal array recorder, Nihon Kohden, Tokyo, Japan). Benidipine was dissolved in saline and was administered intravenously at a dose of $1,3,10$ or 30 $\mathrm{mg} / \mathrm{kg}$. The duration for each administration was $20 \mathrm{~min}$. Fifteen minutes after each administration, blood (200 $\mu \mathrm{l})$ was collected from the right femoral artery. Plasma samples were collected and treated as described previously, ${ }^{5)}$ and plasma NE and NMN were determined using HPLC-peroxyoxalate chemiluminescence detection system developed by Tsunoda et $a l^{7,8)}$

Animal experiment and analysis of $N E$ and NMN in plasma after administration of SAMe. Four SHR and WKY rats (male, 20-23 week-old) were anesthetized with pentobarbital (50 mg/kg, i.p.), and the right carotid artery and the jugular vein were catheterized with each polyethylene cannulae. Arterial blood pressure was measured through the right carotid artery

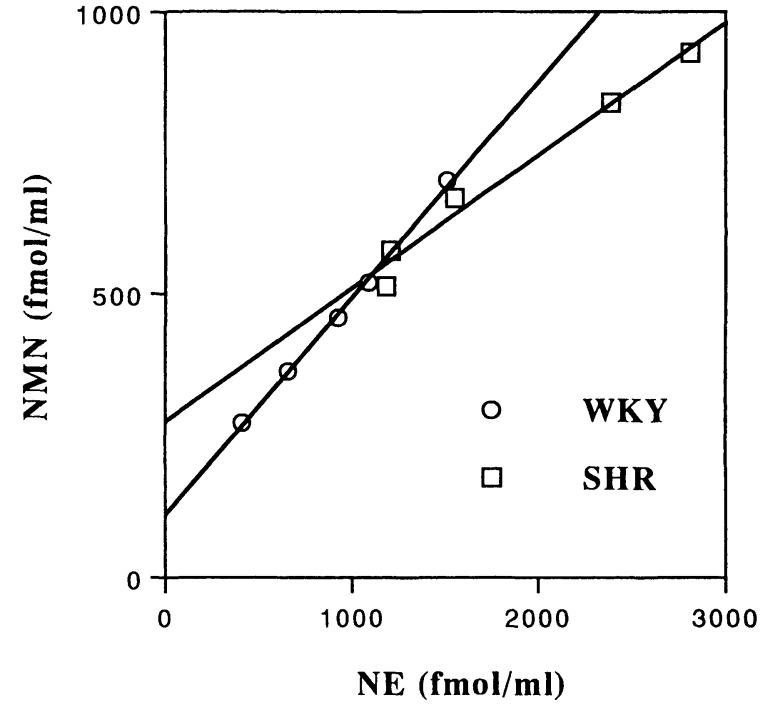

Fig. 1. Relationship between plasma norepinephrine (NE) and plasma normetanephrine (NMN) concentration in the face of an acute hypotension. The equations for SHR ( $\square$ ) and WKY rats $(\bigcirc)$ were $[\mathrm{NMN}]=0.236 \times[\mathrm{NE}]+274\left(\mathrm{r}^{2}=0.978\right),[\mathrm{NMN}]=0.384$ $\times[\mathrm{NE}]+110\left(\mathrm{r}^{2}=0.998\right)$, respectively. The slope for WKY rats was significantly greater than that for SHR $(p<0.05)$.

with cardiac-tachometer. Saline and SAMe solutions were administered through the catheter implanted into the jugular vein at $0 \mathrm{~min}$ and $15 \mathrm{~min}$, respectively. Blood was collected at $-1 \mathrm{~min}, 10 \mathrm{~min}$ and $25 \mathrm{~min}$, and plasma NE and NMN were determined by the same methods as described previously. ${ }^{7), 8)}$

Results and discussion. In an untreated state, the concentrations of the plasma NMN as well as plasma NE were significantly higher in SHR than in WKY rats (Table I), whereas the ratio of plasma NMN to plasma NE was not different between the two strains. In addition, we have found that SHR had a higher erythrocyte COMT activity than WKY rats $(9.28 \pm 0.50$ vs. $6.89 \pm 0.34$ $\mathrm{pmol} / \mathrm{min} / \mathrm{mg}$ protein, respectively, $\mathrm{p}<0.001, \mathrm{n}=5$ ). Previously, Trajkov et al. also reported, compared to WKY rats, SHR had a higher COMT activity in blood vessels and thus an enhanced methylation activity. ${ }^{9)}$ These data indicated that the methylation activity in SHR is activated in an untreated state.

In an acute hypotension, when the blood pressure was reduced by benidipine, however, the ratio of NMN to NE in plasma was lower in SHR than in WKY rats (Fig. 1). These data indicate that, in an induced hypotension where NE rapidly increases, the extraneuronal inactivation of the released NE to NMN is decreased. This suggests that the activity of COMT is blunted in SHR as compared to WKY rats. However, we, as well as others, 
(a)

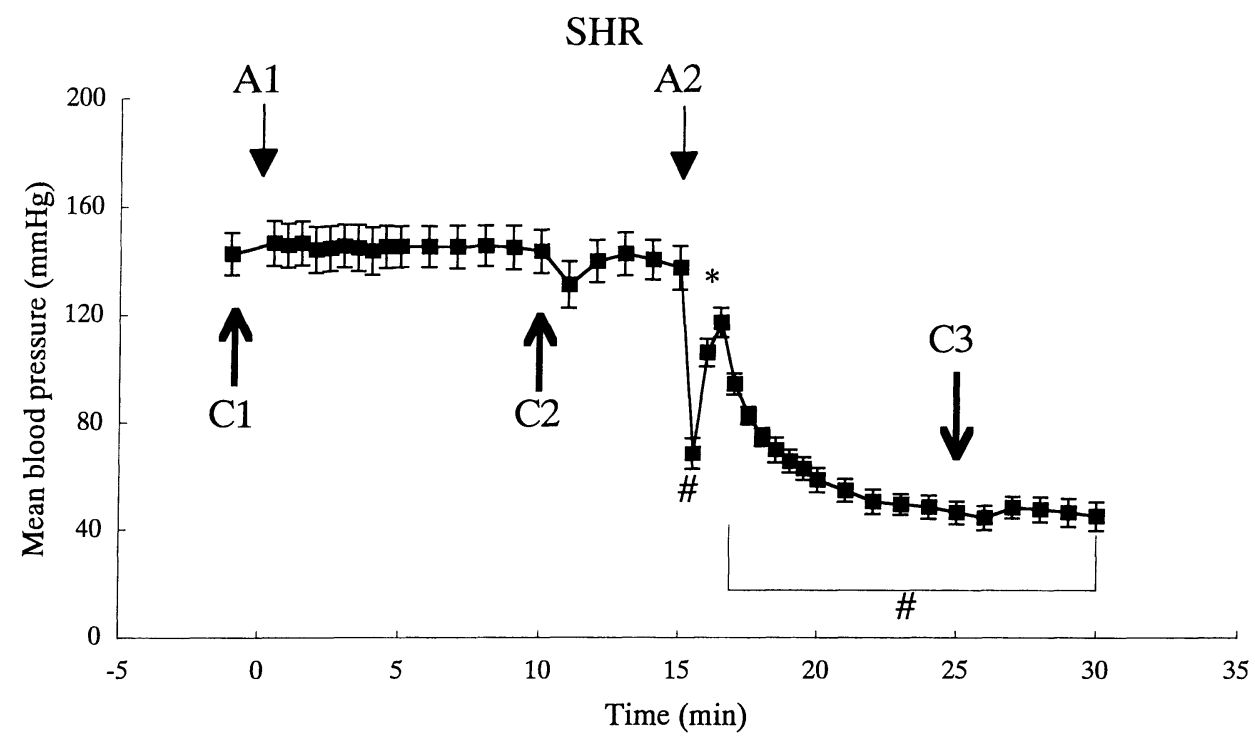

(b)

WKY

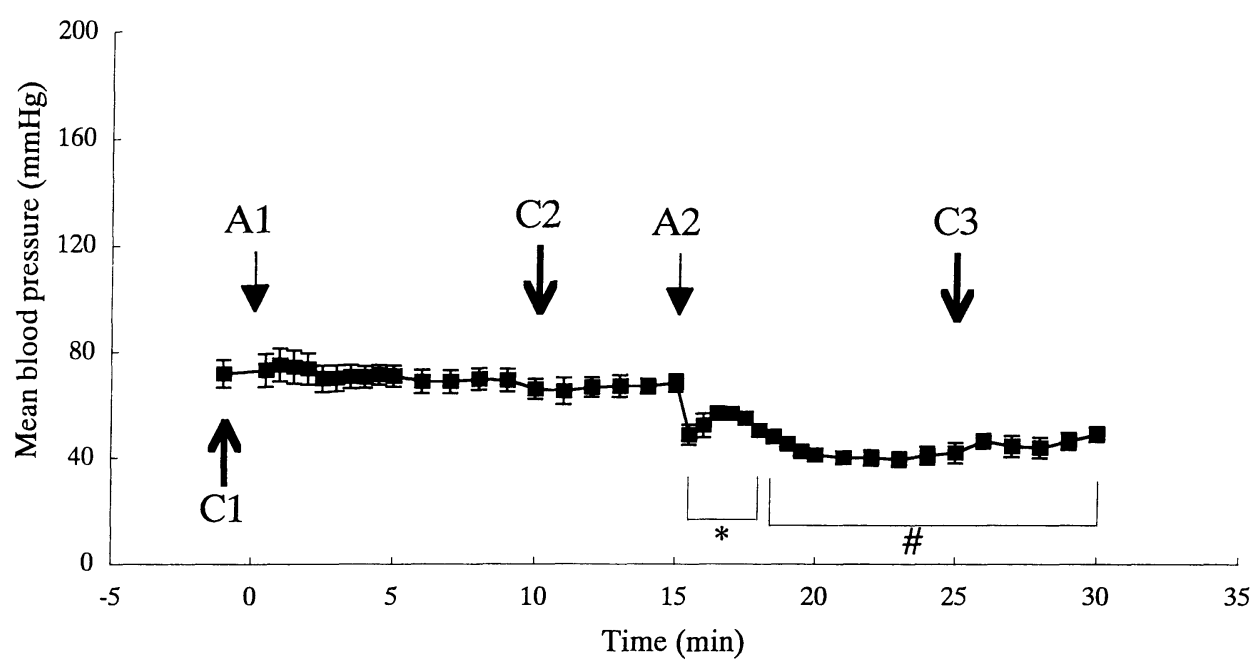

Fig. 2. Changes of mean arterial blood pressure after the intravenous administration of S-adenosyl-L-methionine (SAMe) at a dose of 1.0 $\mathrm{mg} / \mathrm{kg}$ (dissolved in saline, injected at a volume of $1.0 \mathrm{~mL} / \mathrm{kg}$ ) or saline $(1.0 \mathrm{~mL} / \mathrm{kg}$ ) to SHR (a) or WKY rats (b). The results represent the mean \pm SEM. Saline and SAMe solutions were administered through the catheter implanted into the jugular vein at $0 \mathrm{~min}(\mathrm{Al})$ and $15 \mathrm{~min}$ (A2), respectively. Blood was collected at $-1 \mathrm{~min}(\mathrm{C} 1), 10 \mathrm{~min}(\mathrm{C} 2)$ and $25 \mathrm{~min}(\mathrm{C} 3)$. *: $\mathrm{p}<0.05$, \#: $\mathrm{p}<0.01$ vs. the values at $-1 \mathrm{~min}$.

have found that, in in vitro experiments, SHR had a higher COMT activity than WKY rats. At the moment, we are not able to satisfactorily explain the discrepancy, for which further studies are required.

Since the activation of COMT could further methylate NE to NMN and thus reduces the blood pressure, we have studied the balance of COMT and its coenzyme and cofactor, such as SAMe and magnesium, or its inhibitors such as S-adenosyl-L-homocysteine ${ }^{10)}$ in their effects on blood pressure. At present, although we have no agent known to activate COMT, we have selected SAMe to activate the enzyme and examined its effects on blood pressure. We administered a single dose of SAMe and examined the relationships between hypotension and plasma NE or plasma NMN. Intravenous administration of SAMe at $1.0 \mathrm{mg} / \mathrm{kg}$ induced a significant hypotensive response (Fig. 2a), a significant reduction of plasma NE concentration, and a significant increase of plasma NMN concentration in SHR (Table II). These results suggest that the deficient COMT of SHR may be enhanced by SAMe and hence, a significant reduction of the plasma NE concentration. As can be expected, blood pressure was greatly reduced. Therefore, even though the rate of release of NE into the blood stream is 
Table II. Effects of SAMe on mean arterial blood pressure, plasma norepinephrine (NE) and normetanephrine (NMN) concentration

\begin{tabular}{lccc}
\hline & Pre-treatment $^{\mathrm{a})}$ & Saline $1 \mathrm{~mL} / \mathrm{kg}^{\mathrm{b})}$ & SAMe $1.0 \mathrm{mg} / \mathrm{kg}^{\mathrm{c}}$ \\
\hline WKY(n=4) & & & \\
Blood pressure (mmHg) & $71.8 \pm 5.3$ & $65.9 \pm 3.8$ & $41.9 \pm 3.9^{\#}$ \\
Plasma NE conc. (fmol/ml) & $720 \pm 133$ & $590 \pm 124$ & $766 \pm 75$ \\
Plasma NMN conc. (fmol/ml) & $236 \pm 53$ & $141 \pm 42$ & $316 \pm 35$ \\
SHR (n=4) & & & \\
Blood pressure (mmHg) & $143 \pm 8$ & $144 \pm 8$ & $46.3 \pm 4.3^{\#}$ \\
Plasma NE conc. (fmol/ml) & $1520 \pm 13$ & $1470 \pm 230$ & $1100 \pm 110^{*}$ \\
Plasma NMN conc. (fmol/ml) & $679 \pm 106$ & $962 \pm 135$ & $1150 \pm 110^{*}$ \\
\hline
\end{tabular}

The values represent the mean \pm SEM of 4 rats per group. ${ }^{\text {a), b), c) }}$ : measured at the points corresponding to $\mathrm{C} 1, \mathrm{C} 2$ and $\mathrm{C} 3$ in Fig. 2, respectively. ${ }^{*}: \mathrm{p}<0.05,{ }^{\#}: \mathrm{p}<0.01$ vs. the pre-treatment values.

attenuated in hypertensive state, ${ }^{4), 5)}$ because of the blunted metabolism of $\mathrm{NE}$, the plasma NE remains in a concentration high enough to cause hypertension in animals. When the blunted COMT activity is restored by its coenzyme administration, the decrease of $\mathrm{NE}$ leads to a hypotensive response. Although the hypotensive effect on WKY rats was significant (Fig. 2b), the decrease of NE and the increase of NMN were not (Table II). These data indicate that, in WKY rats, a small but enhanced methylation occurred to reduce the extraneuronal NE followed by a significant reduction of blood pressure. However, a rapid recovery of the release of $\mathrm{NE}$ would have maintained the concentration of plasma NE. A more detailed examination should be required including the time course of the release and methylation of NE induced by the blood pressure reduction by SAMe in both strains of rats. It is of interest to note that the blood pressure responses to SAMe were biphasic in both SHR and WKY rats. The reasons for the biphasic response (Fig. 2) remain to be elucidated.

In conclusion, we report, for the first time, a coenzyme of COMT, SAMe, was found to decrease blood pressure in hypertensive rats. The finding raised a possibility that coenzymes of COMT may be useful agents for the treatment of hypertension. Since SAMe is now available in Europe as a remedy for neurological disorder and liver disease ${ }^{10), 11)}$ we propose that SAMe be evaluated as a remedy for human hypertension.

Acknowledgements. We thank Dr. C. Lee of Yamato Scientific for critical and helpful comments and reviews.

\section{References}

1) Imai, K. (1998) Anal. Sci. 14, 257-264.

2) Higashidate, S., Imai, K., Prados, P., Adachi-Akahane, S., and Nagao, T. (1994) Biomed. Chromatogr. 8, 19-21.

3) Imai, K., Higashidate, S., Prados, P., Santa, T., AdachiAkahane, S., and Nagao, T. (1994) Biol. Pharm. Bull. 17 , 907-910.

4) Prados, P., Santa, T., Homma, H., Doi, H., Narita, H., Castillo, B. D., Martin, M. A., and Imai, K. (1997) Biol. Pharm. Bull. 20 341-344

5) Prados, P., Santa, T., Fukushima, T., Homma, H., Kasai, C., Martin, M. A., Castillo, B. D., and Imai, K. (1998) Hypertens. Res. 21, 147-153.

6) Shultz, E., Nissinen, E., and Kaakkola, S. (1989) Biomed. Chromatogr. 3, 64-67.

7) Tsunoda, M., Takezawa, K., Santa, T., and Imai, K. (1999) Anal. Biochem. 269, 386-392.

8) Prados, P., Higashidate, S., and Imai, K. (1994) Biomed. Chromatogr. 8, 1-8.

9) Trajkov, T., Berkowitz, B. A., and Spector, S. (1974) Blood Vessels. 11, 101-109.

10) Bottiglieri, T., Hyland, K., and Reynolds, E. H. (1994) Drugs. 48, 137-152.

11) Lieber, C. S., and Williams, R. (1990) Drugs. 40, 1-138.

12) Vlachakis, N. D., Alexander, N., and Maronde, R. F. (1980) Clin. Exp. Hypertension. 2, 309-319. 